\title{
Evaluación del uso de las competencias laborales en la industria de la contrucción en el Distrito Federal
}

\author{
Evaluation of the use of skills work in construction industry in Mexico city
}

Bernabé Martínez

\section{Resumen}

El propósito de trabajo consiste en determinar la influencia de la Gestión por Competencias laborales utilizadas en la industria de la construcción en la ciudad de México, mediante la aplicación de un instrumento construido ex profeso, con el propósito de conocer las de mayor presencia en el sector, a fin de que puedan ser aprovechadas por las micro, pequeñas y medianas compañías constructoras del Distrito Federal, a efecto de incrementar su productividad y participación económica en la ciudad.

\begin{tabular}{|c|c|}
\hline $\begin{array}{l}\text { Palabras claves } \\
\text { - Producción y estructura del mercado } \\
\text { - Politica industrial } \\
\text { - Formación de capital humano }\end{array}$ & $\begin{array}{l}\text { Key words } \\
\text { - Production and market structure } \\
\text { - Economies of regulation } \\
\text { - Human capital formation }\end{array}$ \\
\hline \multicolumn{2}{|c|}{ JEL: LI I, L52,J24 } \\
\hline
\end{tabular}

\section{Introducción}

En términos generales, puede afirmarse que directivos y responsables de los proyectos y obras desarrolladas por empresas de la industria de la construcción en el Distrito Federal, menosprecian las actividades administrativas. La aplicación de técnicas concebidas para obtener mayor productividad del personal ocupado es tangencial, por no decir nula. La mayoría de las constructoras omiten utilizarlas. A pesar de que el manejo adecuado y oportuno del factor humano tiende a garantizar mayor productividad en la ejecución de sus actividades y mejor aun, permite acrecentar la utilidad de las empresas.

La gestión de recursos humanos por competencias laborales es un instrumento orientado a propiciar mayor productividad y utilidad para las empresas. Es una nueva técnica de trabajo usada por organizaciones mundiales' para obtener mejores resultados en su operación; misma que puede ser utilizada de manera permanente por la industria de la construcción. Las preguntas que debe responder para evaluar la posibilidad de su uso en el sector, consiste en determinar: ¿Hasta qué punto empresas constructoras del Distrito Federal, utilizan competencias laborales en la realización de sus trabajos?, ¿con qué grado de profundidad las aplican en la ejecución de sus actividades? ¿Es posible encontrar las competencias de mayor uso, que permitan definir un modelo básico susceptible de ser implantado por las micro, pequeñas y medianas empresas de esta industria? Y como sector ¿Qué impacto económico

I Organización Internacional del Trabajo. CINTERFOR. Proyecto de competencias laborales, "Análisis de la experiencia comparada:Australia, Canadá, Francia, Gran Bretaña, México”, página web, 27 de septiembre del 2000. 
tienen estas empresas por no aplicar el uso de esta técnica de evaluación? Esas interrogantes, pretende responder el presente trabajo, por demás tema neural de la investigación. La pretensión académica se finca en identificar las competencias que utiliza la industria de la construcción, con el propósito de precisar las de mayor presencia en las compañías del ramo, para que micro, pequeñas y medianas compañías del sector del Distrito Federal, puedan implantarlas en sus respectivas empresas. El instrumento dispuesto para su medición fue una encuesta construida ex profeso. Se enfocó a buscar cuáles eran las competencias más representativas del sector para el 2008, pero que tiene vigencia para el año 20I2, con el fin de proponerlas como posible alternativa de solución en la búsqueda del incremento de la productividad empresarial. Además, permite evaluar si las empresas entrevistadas tienen conciencia sobre la utilidad de las competencias en el desarrollo de sus actividades, así como el grado de uso de la gestión estratégica por competencias laborales en la industria de la construcción en esta entidad.

Los resultados del estudio indican el grado de profundidad relativo del uso de las competencias laborales y permiten sugerir las doce competencias ubicadas para la industria de la construcción y utilización en la práctica.Además, se muestran las gráficas que describen y avalan la inferencia determinada sobre el entorno que rodea las competencias motivo de la investigación.

Finalmente, se presentan las conclusiones originadas por el estudio y las propuesta de aplicación de competencias laborales en la industria, basada en los resultados del estudio que pueden utilizarse en las micro, pequeñas y medianas empresas constructoras, para incrementar su productividad y, en consecuencia sus ingresos.

\section{Acercamiento a las competencias laborales}

En la actualidad, se habla de competencias básicas, competencias de ciudadanía o competencias para la integración social.' Todo individuo debería adquirir estas competencias para participar en los diversos ámbitos de socialización, tales como la familia, la comunidad, la escuela, el trabajo, la práctica de deportes; utilizar el tiempo libre con otras personas o eventos de orden social, cultural o esparcimiento.Allí, las personas adquieren reglas de acción, modos de relación y comunicación, formas de pensamiento lógico matemático, reconocimiento de expectativas de otros y maneras de ser con respecto al otro.

A partir de las denominadas competencias básicas, las competencias laborales presuponen su desarrollo, debido a que han permitido desenvolverse, profundizarse y especificarse en modos profesionales. Por esta razón, cuando las sociedades excluyen a grandes segmentos de su población del mercado de trabajo, bloquean 
ámbitos importantes del desarrollo y fortalecimiento de competencias básicas y competencias técnicas.

El conocimiento de cómo especificar una competencia laboral es trascendente para las empresas. Por tanto, debe conversarse con los trabajadores que ejercen competencias como parte de su profesión cotidiana. Normalmente, las personas trabajan y se desenvuelven en distintos roles laborales. Rara vez se detienen a reflexionar sobre lo qué hacen, cómo lo hacen y cómo se dan cuenta de que obran bien y con calidad, en condiciones seguras de trabajo.

De lo anterior, puede inferirse que una competencia tiene mayor nivel que una habilidad, debido a que integra un conjunto de habilidades, conocimientos y comportamientos que subyacen en el individuo para desempeñar con éxito una actividad dada. Dicho de otro modo una competencia integra: saber, saber hacer, saber ser, querer hacer y poder hacer. ${ }^{2}$ Para que una persona muestre estos comportamientos que integran las competencias se hace necesaria la definición y unión de estos cinco elementos fundamentales:

a) Saber: conjunto de conocimientos relacionados con los comportamientos implicados en la competencia. Pueden ser de carácter técnico y de carácter social. En ambos casos la experiencia juega un papel esencial.

b) Saber hacer: conjunto de habilidades que permiten poner en práctica los conocimientos que se poseen. Puede hablarse de habilidades técnicas, sociales y cognitivas; por regla general deben interactuar entre sí.

c) Saber ser: conjunto de actitudes acordes con las principales características del entorno organizacional o social. Es decir, se trata de tener en cuenta los valores, creencias y actitudes como elementos que favorecen o dificultan determinado comportamiento en un contexto dado.

d) Querer hacer: conjunto de aspectos motivacionales responsables de que la persona quiera o no realizar los comportamientos propios de la competencia. Se tratan de factores de carácter interno o externo a la persona, que determinan un esfuerzo o no, por mostrar una competencia.

e) Poder hacer: conjunto de factores relacionados con dos cuestiones fundamentales: lo individual y lo situacional. El primer punto de vista se refiere a la capacidad personal, es decir, las aptitudes y rasgos personales considerados como potencialidades de la persona. $Y$ desde el punto de vista situacional, comprende el grado de "favorabilidad" del medio, es decir, diferentes situaciones pueden marcar distintos grados de dificultad para mostrar un

2 Fernández, A. M. “Pensemos en las Competencias”, disponible en:www.gestiopolis.com/canales7/rrhh/competencias-competentes y competitividad.htmUTH (2006). 
comportamiento dado. Es válido destacar la disponibilidad o no de medios y recursos que faciliten o dificulten el desempeño de la competencia.

En su conjunto conducen a la competencia que resulta observable para los demás y que permite establecer diferentes niveles de desempeño de las personas en su ámbito personal o profesional, ya sea durante la realización de tareas diversas o en sus interacciones sociales. Por tanto, la competencia puede considerare como un nuevo paradigma del empleo. Los atributos que debe tener un trabajador para ocupar un determinado puesto. En las actuales condiciones de competitividad y productividad el concepto de competencia hace referencia a la formación y desarrollo del capital humano. El concepto competencia busca definir y evaluar las capacidades del trabajador según su desempeño en una situación de trabajo. La competencia laboral no es una probabilidad de éxito en la ejecución del trabajo, es una capacidad real y demostrada. ${ }^{3}$ El concepto de competencias ha sido un dilema para muchas empresas. El querer implementar tal concepto ha llevado a los directivos empresariales y consultores organizacionales a analizar detalladamente la teoría y diseñar estrategias que permitan estructurar una propuesta metodológica para el diseño de un modelo de competencias laborales al interior de cada organización.

Las competencias pueden caracterizarse de manara individual o grupal dentro de las organizaciones. Existen diferentes Modelos, entre los más utilizados se encuentran: el Modelo Conductista, El Modelo Funcionalista y El Modelo Constructivista. ${ }^{4}$ Ahí pueden inscribirse el conglomerado de definiciones que intentan otorgarle precisión a la competencia:

a) Modelo Conductista: orientado a la selección de los "más aptos" y portador de dificultades para la identificación de los estándares y requerimientos técnicos. Está basado en los aspectos personales del desempeño laboral susceptibles de ser medidos. Aunque contenga elementos de trabajo colectivo, el trabajador se ve incitado a la superación individual. En este modelo la "competencia" describe fundamentalmente lo que un trabajador "puede" hacer y no "lo que hace". Se centra en la identificación de las capacidades de la persona que la motivan a buscar desempeños superiores en la organización. Generalmente se aplica a los niveles directivos y se suscribe a las capacidades que le permiten destacar en circunstancias no predefinidas.

3 Donna. G., Cuando es imprescindible generar empleabilidad. Empresas y trabajadores en búsqueda de formación por competencias. Página Web.Argentina, 2003 http://www.psiconet.com.

4 Quesada Martínez, Humberto, Competencias Laborales: la puesta en valor del Capital Humano, http://www.sht. com.ar/archivo/temas/competencias2.htm 
b) Modelo Funcionalista: basado esencialmente en la medición de resultados. Parte del objetivo principal y desagrega en niveles hasta identificar las unidades de competencias y sus elementos. La característica fundamental de este análisis es que describe productos, no procesos. Con este método el trabajador obtiene reconocimiento de sus competencias respecto a la consecución del objetivo principal. La aproximación funcional se refiere a desempeños concretos y predefinidos que la persona debe demostrar, derivados de un análisis de las funciones que componen el proceso productivo. Generalmente, se usa a nivel operativo $y$, se circunscribe a aspectos técnicos. Algunos ejemplos de esta definición son:

c) Modelo Constructivista: es el método con mayor carga holística, con parámetros de inclusión social y formación integral, dimensión humana de la actividad y contexto social del trabajo. Se parte de la premisa de que la participación plena de los individuos en la discusión y comprensión de problemas resulta crucial para la identificación de las "disfunciones" en la empresa. A partir de esa discusión e intercambio general comienza a generarse la norma o estándar. Por ejemplo, una empresa hace conciencia entre todo el personal de que no se tienen definidas las rutinas de mantenimiento preventivo, ni las técnicas para el predictivo. A la vez que se diseñan estas rutinas y técnicas, las competencias del personal implicado van emergiendo.

Derivado de lo anterior, puede afirmarse que la competencia es la capacidad efectiva para llevar a cabo exitosamente una actividad laboral plenamente identificada, así como desempeños satisfactorios en situaciones reales de trabajo, que subyacen en el individuo, en el marco cultural y social de la organización. Se forman a partir del desarrollo de un pensamiento científico-técnico reflexivo, para estar en posibilidad de construir marcos referenciales de acción aplicables a la toma de decisiones que exigen los contextos profesionales para desarrollar y asumir actitudes, habilidades y valores compatibles con las decisiones que deben tomarse cotidianamente, en los procesos en donde debe actuarse responsablemente para obtener determinados productos. En tal sentido no es una probabilidad de éxito en la ejecución de un trabajo; sino una capacidad real y demostrada, al tomar en cuenta además del aspecto cognitivo, también el emocional, que pueden observarse en una situación cotidiana de trabajo. 


\section{Competencias laborales y generación de valor}

Proponer la implantación de competencias en el entorno de la Gestión del Capital Humano, equivale hablar de la gestión del talento. ${ }^{5}$ El mercado concede un indudable valor a la capacidad de innovación de las empresas. Más que sus activos tangibles, evalúa los activos intangibles. Se constata entonces que el valor de mercado difiere habitualmente del valor en libros, debido a la capacidad de predicción que tienen los intangibles de una empresa. En cálculos efectuados recientemente por el Banco Mundial, en relación a los 29 países que concentran $80 \%$ de la riqueza del planeta, se estimó que deben su bienestar en $67 \%$ al capital humano o intelectual, lo que evidencia por qué se hace necesario tenerlo identificado y contabilizado de algún modo para que pueda ser gestionado. ${ }^{6}$ Cuando se crea valor como objetivo permanente y sostenido debe tenerse en cuenta el aprendizaje organizacional, derivado del establecimiento de las estructuras y procedimientos que favorezcan la adquisición y acumulación de conocimiento. Se trata de garantizar que al momento de utilizarlo, sea capaz de transformar una entrada (información, material) en una salida con valor añadido.

Al segmentar el conocimiento se está en mejores condiciones de determinar la contribución del valor creado o por crear; es decir, el éxito de la estrategia de una empresa dependerá de la contribución en la creación de valor de cada miembro de la organización. Para que mantenga una característica sostenida debe considerar una válida y fiable evaluación de la amplitud y profundidad que cada miembro de la organización posee en cada competencia conductual.

Una inversión en tiempo y esfuerzos de los jefes y mandos en el desarrollo de sus colaboradores tiene que considerarse como una inversión financiera. De cualquier manera el conocimiento es generador de riqueza y recae en las personas, quienes son la unidad organizativa básica de la empresa cuya evaluación se realiza no solo en función de lo que saben (conocimientos), sino, y sobre todo, en lo que saben hacer (habilidades), saben ser (actitudes), quieren hacer (motivaciones) y pueden hacer (capacidades).

De esta manera pueden definirse algunos términos que con frecuencia se utilizan en la empresa, ${ }^{7}$ relativos al valor que pueden generar:

5 Evis Contreras Escobar. Recursos Humanos: fuente de inagotable valor. Serie:Tras la esencia vital de la empresa.

Centro de Información. Cubalse. p. 19.

6 Arce, Enrique. www.portaldelconocimiento.com Lecturas - Fichas. Temas: Gestión por Competencias. Artículo: Competencias. Contribución a la creación de valor a través del conocimiento.

7 En el ámbito de la empresa es común utilizar conceptos ligados a la generación de valor entre los que se encuentra Adalberto Chiavenato, Introducción a la Teoría General de la Administración, Mc Graw-Hill/Interamericana de México, S. A. de C.V., Séptima Edición, Tlalnepantla, Estado de México, 
Una cuestión crítica que atiende el modelo, estriba en impulsar la innovación para el liderazgo tecnológico, en virtud de que los trabajadores conocerán su propio perfil de competencia y el requerido por el puesto que ocupan o aspiran ocupar, al identificar y actuar en acciones tendientes a conseguir el perfil requerido. Se incentiva así, el clima innovador desde la base, fundamentalmente a través del auto desarrollo. Ello permite profundizar la alianza estratégica empresa - trabajador al generar mejores recursos humanos, con desarrollo de carrera, movilidad y flexibilidad. Por tanto, ayuda a realizar proyectos empresariales de bajo costo y alto VAN, mediante la utilización de los mejores recursos humanos de la empresa, al distribuirlos según las necesidades de cada proyecto y permite la capitalización de sus experiencias y conocimientos.

\section{Competencias en el ámbito de estudio}

Las competencias generales, son aquellas que abarcan a casi todas las profesiones y oficios; están relacionadas con la preparación básica del individuo. Por ejemplo, utilizar la computadora, comunicarse oral y escrito en su idioma, interpretar texto en una lengua extranjera, aplicar métodos de cálculo, etc. Mientras que las competencias particulares o específicas, son las que determinan la profesión, por ejemplo, elaborar un medicamento, programar determinado tipo de software, realizar determinado tipo de operación quirúrgica. No obstante, también hay otra clasificación consistente en básicas, genéricas y específicas. Un diseño por competencias laborales radica en estructurar didácticamente lo que un individuo necesita saber, saber hacer, saber estar, querer hacer y poder hacer, según las normas de la profesión para la que se está formando. Las competencias utilizadas en el estudio se ciñeron a la clasificación desarrollada por Marta Alicia Alles, ${ }^{8}$ las cuales esencialmente son las siguientes:

\section{Resultados del estudio}

En el 2008, el Distrito Federal contaba 523 empresas constructoras registradas en el padrón del Sistema de Información Empresarial Mexicano (SIEM), de las cuales obtuvo una muestra de 63 organizaciones susceptibles de aplicarles el instrumento desarrollado sobre el particular.

2007, Reyes Ponce, entre otros.

8 Alles, Martha Alicia, Gestión por Competencias, El diccionario, editorial Granica, Buenos Aires, Argentina, 2007. pp. 105-126. 
La encuesta se aplicó entre los meses de abril y de septiembre de 2008. De las compañías contactadas, hubo 47 empresas que pusieron obstáculos al momento de responder el instrumento de medición, de las cuales 41 emitieron evasivas de diversa índole para no conceder entrevistas o alargar el tiempo de espera y 6 de plano no quisieron contestar. Por tanto, existió una negativa tácita en la intención de contestar los reactivos. La desconfianza implícita de la resistencia supone el mal uso que pudiera darse a la información y, al mismo tiempo, refleja la escasa cultura que muestran para ser consultados con fines académicos, en aras de mejorar el desempeño del sector de la construcción.

Por otro lado, al evaluar las 63 encuestas en poder del autor, de empresas que si respondieron, el fenómeno se agranda y se deduce que el $47 \%$ de las entidades vinculadas a la industria, tienen resquemores de todo tipo. Aluden pretextos diversos, como el hecho de argumentar la ausencia de los responsables de generar la información solicitada, no contar con los datos requeridos y en algunos casos, señalaban que eran en extremo confidenciales para exponerlos. Signo inequívoco que pone de manifiesto la poca solidaridad hacia la industria para compartir sus éxitos logrados, o en su caso, exponer las posibles deficiencias de cada organización.

\section{Clasificación}

Las empresas constructoras entrevistadas esencialmente se dedican a la construcción $(28.57 \%)$ y vivienda $(25.40 \%)$ en general; en menor medida al mantenimiento (9.52\%), así como al diseño y construcción (4.8\%), que en su conjunto representan más de $73 \%$ de las actividades desarrolladas por las empresas. Al desagregarlas se infiere, de acuerdo al instrumento de medición, que dedican a diversidad de tareas vinculadas a la industria y no a una actividad específica, no están especializadas. Llama la atención la falta especialización debido a que desarrollan sus actividades en cuando menos dos áreas de la construcción. Eso hace suponer que son de sobrevivencia porque trabajan en "lo que sea" y no en una tarea preponderante, como debiera ser vivienda, acabados, caminos. 


\section{Cuadro I}

Distribución por área de las empresas de construcción

en el Distrito Federal

\begin{tabular}{|l|c|c|}
\hline \multicolumn{1}{|c|}{ Área } & Cantidad & $\%$ \\
\hline Acabados & 4 & 6.35 \\
\hline Construcción & 18 & 28.57 \\
\hline Vivienda & 16 & 25.40 \\
\hline Mantenimiento & 6 & 9.52 \\
\hline Proyectos & 4 & 6.35 \\
\hline Supervisión & 2 & 3.17 \\
\hline Diseño y construcción & 6 & 9.52 \\
\hline Vías y caminos & 4 & 6.35 \\
\hline Construcción de aeropuertos & 1 & 1.59 \\
\hline Petroleras & 1 & 1.59 \\
\hline Pinturas & 1 & 1.59 \\
\hline & 63 & 100.00 \\
\hline
\end{tabular}

Fuente: Identificación de Competencias Laborales en la industria de la construcción (2008), encuesta elaborada por el autor.

El principal puesto de la persona que atendió la encuesta fue el gerente (19.05\%), le siguió el administrador (14.3\%), director y dueño (9.52\%), gerente general y residente (6.35\%). En su conjunto abarcan más de $65 \%$ de la muestra. Ello permite inferir que quienes contestaron el instrumento son personas que toman decisiones en la organización, y en términos generales a quienes se dirigió el estudio. Las persona de menor nivel que atendieron la encuesta fueron: ventas $(4.8 \%)$ y un instalado -dueño (I.6\%). Al sumar el conjunto de los cuestionarios se significa la importancia de la investigación, en virtud de que casi $94 \%$ de los consultados tiene en mayor o menor medida, algún cargo de dirección y por ende, tiene la consciencia suficiente sobre la intención de la indagación y un panorama amplió sobre las actividades administrativas desarrolladas en cada empresa; es decir, es personal que garantiza la veracidad de la información y por tanto, de los resultados obtenidos. 


\section{Cuadro 2}

Integración de puestos directivos en la Industria de la Construcción en el Distrito Federal

\begin{tabular}{|l|l|l|}
\hline \multicolumn{1}{|c|}{ Directivo } & Cantidad. & \multicolumn{1}{c|}{$\%$} \\
\hline Supervisor & 2 & 3.17 \\
\hline Residente & 4 & 6.35 \\
\hline Jefe de Proyecto & 3 & 4.76 \\
\hline Instalador Dueño & 4 & 6.35 \\
\hline Gerente y Diseño & 2 & 3.17 \\
\hline Gerente General & 4 & 6.35 \\
\hline Gerente de Construcción & 3 & 4.76 \\
\hline Gerente & 12 & 19.06 \\
\hline Encargado & 6 & 9.52 \\
\hline Director Jurídico & 3 & 4.76 \\
\hline Director & 6 & 9.52 \\
\hline Desarrollador & 3 & 4.76 \\
\hline Contador General & 2 & 3.17 \\
\hline Administrador & 9 & 14.30 \\
\hline Total & 63 & 100.00 \\
\hline
\end{tabular}

Fuente: Identificación de Competencias Laborales en la industria de la construcción (2008), encuesta elaborada por el autor.

\section{Competencias gerenciales}

Liderazgo: Es la capacidad de dirigir a un grupo o equipo de trabajo del cual dependen otros equipos. Implica el deseo de guiar a los demás. Crea un clima de energía y compromiso; comunica la visión de la empresa, tanto desde una posición formal como desde una informal de autoridad. En sentido amplio el equipo debe considerarse como cualquier grupo en el que la persona asume el papel de líder.

Empoderamiento: establece objetivos claros de desempeño y las responsabilidades personales. Proporciona dirección y aprovecha la heterogeneidad de los miembros del equipo para agregar un valor añadido a la empresa. Combinan adecuadamente situaciones, personas y tiempos. Comparte las consecuencias de los resultados. Emprende acciones para mejorar el talento y las capacidades de los demás.

Desarrollo del equipo: es la habilidad para desarrollar el equipo humano hacia adentro; facilidad para relacionarse interpersonalmente y la capacidad de comprender la repercusión que las acciones personales sobre el éxito de las acciones de los demás. Incluye también la capacidad de crear adhesión, compromiso y fidelidad. 
Trabajo en equipo: se fundamenta en la capacidad de colaborar y cooperar con los demás, formar parte de un grupo y trabajar juntos; con una actitud genuina. Es conveniente que el ocupante del puesto sea un miembro de un grupo que funcione en equipo. Equipo en su definición más amplia, entendido como un grupo de personas que trabaja en procesos, tareas u objetivos compartidos.

\section{Competencias de logro y acción}

- Dinamismo-energía o adaptabilidad: es la facilidad para trabajar duro en situaciones cambiantes o alternativas, con interlocutores diversos, que cambian en cortos periodos de tiempo, en jornadas de trabajo prolongadas, sin que se vea afectada su actividad.

- Iniciativa: es la predisposición a actuar proactivamente y a pensar en lo que hay que hacer en el futuro, involucra marcar el rumbo de mediante acciones concretas, no únicamente de palabra. Los niveles de actuación van desde concretar decisiones tomadas en el pasado hasta la búsqueda de nuevas oportunidades o solución de problemas.

- Orientación a resultados: Es la capacidad para actuar con velocidad y sentido de urgencia cuando se deben tomar decisiones importantes y necesarias para superar las metas y los objetivos fijados; responder a las necesidades del cliente o mejorar la empresa. Es la capacidad de administrar los procesos establecidos para que no interfieran con la consecución de los resultados esperados.

- Pensamiento estratégico: habilidad para comprender rápidamente los cambios del entorno, las oportunidades, amenazas, fortalezas y debilidades de la empresa, al momento de identificar la mejor propuesta estratégica.

- Orientación al cliente: reside en el deseo de ayudar o servir a los clientes, de comprender y satisfacer sus necesidades. Se cifra en esforzarse por conocer y resolver problemas del cliente, tanto del cliente final al que van dirigidos los esfuerzos de la empresa, como los clientes de sus clientes y todos aquellos que cooperen en la relación empresa - cliente, como los proveedores y el personal de la institución.

- Modalidad de contacto personal: es la capacidad de demostrar una sólida habilidad de comunicación. Alienta a otros a compartir información, habla con todos y valora las contribuciones de los demás. Sabe escuchar hace posible que los demás tengan fácil acceso a la información que se posee.

- Habilidad para el cambio: es la aptitud para comunicar la visión de la estrategia de la empresa, en donde hace que esa visión parezca no solo posible sino deseable al órgano decidor; es decir, al Consejo de Administración o al dueño de la empresa, 
con capacidad de crearles una motivación y compromiso genuinos, actúa como propulsor de la innovación y genera cambios frecuentes en la organización.

- Integridad: es la capacidad para actuar en consonancia con lo que se dice o se considera importante. Incluye comunicar intenciones, ideas y sentimientos abierta y directamente $y$, estar dispuesto a actuar con honestidad, incluso situaciones difíciles con personas externas. Las acciones son congruentes con lo que dice. Queda fuera el doble discurso entre el ser y el quehacer.

\section{Determinación de las competencias aplicables en el distrito federal}

En el Distrito Federal, cuando se diseñan proyectos o ejecutan edificaciones, con frecuencia se pone énfasis en la evaluación de la capacidad técnica de los profesionales de la construcción y se deja de lado otros aspectos relevantes que inciden directamente en la rentabilidad de las empresas, como es el caso del manejo de los recursos humanos. Las razones argüidas al respecto, son de diversa índole, como saber efectuar cálculos estructurales para soportar el peso de las edificaciones, tratar de cumplir con el plan de obras, entregas oportunas de los proyectos de obra, todas ellas validas, sin duda, sobre todo para quienes son dueños o dirigen las organizaciones en la industria de la construcción. Si se considera que la actual crisis financiera de orden global, impacta a prácticamente a todos los sectores económicos y, en particular a la industria de la construcción, se hace necesario establecer acciones que permitan mantenerse y más aún consolidarse de forma permanente en un mercado cada vez más competido. Significa utilizar al máximo el talento y capacidades del recurso humano. Por eso debe irse más allá, si el propósito se orienta a tener una empresa de calidad y competitiva.

Un aspecto relevante que debe considerar y plantear cualquier empresa, independientemente de que esté ligada a la construcción, radica en determinar cómo conformar $y$, en su caso, transformar al factor humano -entendido como el capital capaz de generar valor agregado en la industria- de acuerdo a las exigencias del entorno. Hay muchos profesionales técnicamente capacitados para desarrollar actividades productivas de toda índole, como las inherentes al diseño y construcción de obras. La duda surge cuando se necesita reconocer y definir las características indispensables de comportamiento medible y observable de éste personal, para el cumplimiento eficaz de sus funciones. De existir algunas carencias, las repercusiones inmediatas derivan en el retraso en la entrega de las edificaciones, proyectos mal calculados, diseños elaborados con deficiencias técnicas, que de inmediato repercuten en la productividad, conducen a la disminución de ingresos y ponen en riesgo la supervivencia de la empresa. 
De ahí que el estudio se enfoque a considerar aristas vinculadas al manejo de la gestión por competencias laborales. Por eso, se pretende identificar las competencias utilizadas en la industria de la construcción, con el propósito de precisar las de mayor presencia para que puedan aprovecharse en las compañías constructoras micro, pequeñas y medianas en el Distrito Federal, mediante la aplicación de una encuesta construida en materia de gestión estratégica de competencias laborales.

Las competencias laborales indicas, son enunciativas más no limitan a la organización a ceñirse únicamente a ellas, debido a que pueden identificarse otras probables que pudieran ser aprovechadas. No obstante, es pertinente señalar que pueden ser las más relevantes que podrían considerar las empresas del sector de la construcción, al diseñar o ejecutar proyectos de obra.

Lo anterior, no significa que a lo largo de la historia se haya carecido de competencias laborales para transformar la naturaleza en edificaciones en beneficio del hombre. No. Han sido utilizadas siempre, subyacen en cada diseño y obra ejecutada. Simplemente, se ha omitido su importancia y real envergadura. Se requiere hacerlas conscientes, ponderarlas y utilizarlas en un mundo cada vez más competido. Hacerlas manifiestas y potenciar su aplicación, podrá generar mayor bienestar en el sector. De modo que, a mayor nivel de conocimiento de las competencias laborales o aplicación en empresas de la industria de la construcción mayor productividad y presencia tendrán en el mercado del Distrito Federal.

\section{Resultados del estudio}

En el 2008, el Distrito Federal contaba 523 empresas constructoras registradas en el padrón del Sistema de Información Empresarial Mexicano (SIEM), de las cuales obtuvo una muestra de 63 organizaciones susceptibles de aplicarles el instrumento desarrollado sobre el particular.

La encuesta se aplicó entre los meses de abril y de septiembre de 2008. De las compañías contactadas, hubo 47 empresas que pusieron obstáculos al momento de responder el instrumento de medición, de las cuales $4 \mathrm{I}$ emitieron evasivas de diversa índole para no conceder entrevistas o alargar el tiempo de espera y 6 de plano no quisieron contestar. Por tanto, existió una negativa tácita en la intención de contestar los reactivos. La desconfianza implícita que implica la resistencia supone el mal uso que pudiera darse a la información y, al mismo tiempo, refleja la escasa cultura que muestran para ser consultados con fines académicos, en aras de mejorar el desempeño del sector de la construcción.

Por otro lado, al evaluar las 63 encuestas en poder del autor, de empresas que si respondieron, el fenómeno se agranda y se deduce que $47 \%$ de las entidades vin- 
culadas a la industria, tienen resquemores de todo tipo. Aluden excusas de diversa índole, como argumentar la ausencia de los responsables de generar la información solicitada, no contar con los datos requeridos y en algunos casos, señalaban que eran en extremo confidenciales, entre otros. Signo inequívoco que pone de manifiesto la poca solidaridad hacia la industria para compartir sus éxitos logrados, o en su caso, exponer las posibles deficiencias de cada organización.

\section{Competencias gerenciales}

Las competencias gerenciales permiten determinar el grado de dirección, control, seguimiento y coordinación de acciones en torno a las actividades realizadas para el cumplimiento de la misión, visión y objetivos de las organizaciones. Al realizar el análisis general de la vertiente que integran estas competencias, se concluye que en su conjunto las ejecutan siempre o frecuentemente casi $70 \%$ de las empresas. Si se desagregan únicamente el $36.51 \%$ refirió aplicarlas siempre, $32.94 \%$ frecuentemente. El significado del primer resultado adquiere importancia en virtud de la escasa importancia dada a la aplicación de las competencias gerenciales en el contorno de las empresas. Preocupa el hecho de conocer que solo cuatro de cada diez unidades de negocio, manifestaron abiertamente utilizar siempre las competencias aludidas, toda vez que son de enorme utilidad para la toma de decisiones. Constituyen competencias laborales con un peso específico para conseguir la eficacia de la organización; por tanto deben ser entendidas, aplicadas a fondo y aprovechadas al máximo por los dueños y directivos de las constructoras. Liderazgo, empoderamiento, trabajo en equipo y desarrollo del equipo conforman la pieza angular en las empresas. Si desean dar pasos firmes para iniciar la transformación de sus organizaciones en entes exitosas, deben acercarse y dimensionar su significado y ámbito de aplicación. Conocer las implicaciones de implantarlas en cada negocio, con la genuina intención de adaptarlas a las estrategias de la empresa. Una acción habitual que garantice el cumplimiento de los planes y propósitos establecidos por la dirección, sin poner objeciones al desarrollo del activo más importe de las constructoras: el factor humano. Ver Cuadro 3. 


\section{Cuadro 3}

Composición de las competencias gerenciales en la industria de la construcción en el Distrito Federal

\begin{tabular}{|l|c|c|}
\hline Concepto & Total & $\%$ \\
\hline Siempre & 92 & $36.5 I$ \\
\hline Frecuentemente & 83 & 32.94 \\
\hline A veces & 49 & 19.44 \\
\hline No es necesario & 26 & 10.32 \\
\hline No sé & 2 & 0.79 \\
\hline Total & 252 & 100.00 \\
\hline
\end{tabular}

Fuente: Identificación de Competencias Laborales en la industria de la construcción (2008), encuesta elaborada por el autor.

\section{Competencia liderazgo}

El liderazgo es la capacidad de incidir en los demás para lograr que la empresa cumpla a cabalidad con los objetivos trazados. Para ello, los directivos deben estar convencidos de que las metas no solamente son posibles sino a todas luces asequibles. Al crear un clima de energía y compromiso para cumplir con la misión y visión establecida, al ser reconocido como referente en el cual confiar para llevar adelante lo que se propone. Esa característica del líder es la permite incidir en el concierto del área donde interactúa.Tener mayor participación en el mercado es el fin principal de toda organización. El líder lo sabe y debe hacer lo posible para acrecentar su contribución en el conjunto de la industria. En el caso de la industria de la construcción contraviene el espíritu de la competencia laboral mencionada, debido a que cae por debajo del promedio observado en el estudio, pues apenas rebasan $52 \%$ de lo que podría considerarse aceptable para incidir en los demás miembros de la empresa. Solo $31.75 \%$, manifestó emplearla siempre y frecuentemente $20.63 \%$. La situación empeora cuando se llega a los niveles más bajos de desagregación: 15.87\% considera que no es necesario realizar esta competencia y algunas veces $31.75 \%$. En términos prácticos significa que dos de cada diez constructoras no utiliza el liderazgo para cumplir sus cometidos institucionales. Ver Cuadro 4. 


\section{Cuadro 4}

Distribución de la competencia liderazgo en la industria de la construcción en el Distrito Federal

\begin{tabular}{|l|c|c|}
\hline \multicolumn{1}{|c|}{ Concepto } & Cantidad & $\%$ \\
\hline Siempre & 20 & 31.75 \\
\hline Frecuentemente & 13 & 20.63 \\
\hline A veces & 20 & 31.75 \\
\hline No es necesario & 10 & 15.87 \\
\hline No sé & 0 & 0.00 \\
\hline Total & 63 & 100.00 \\
\hline
\end{tabular}

Fuente: Identificación de Competencias Laborales en la industria de la construcción (2008), encuesta elaborada por el autor.

\section{Competencia empoderamiento}

Otorgar poder de decisión al equipo de trabajo es importante en la toma de decisiones. Coadyuva en el logro de los objetivos empresariales. El fijarse un punto de llegada para cumplir con la misión y visión institucional, al establecer objetivos claros de desempeño y compromisos personales, con la intención de agregar valor a la empresa. Se centra en las condiciones del ambiente de trabajo relativas a la diversidad de tareas, autonomía, carga de trabajo, soporte de la organización y posición dentro de la empresa. La capacidad de utilizar la heterogeneidad de los miembros del equipo a favor de las constructoras. Su utilización se demuestra en la práctica.Al revisar las encuestas, se determina que aceptablemente contestaron más de $74 \%$. Siempre respondió $36.51 \%$ y frecuentemente 38 . I por ciento.

\section{Cométencia desarrollo del equipo}

Permitir el crecimiento hacia niveles superiores de la organización mediante la capacitación o a través de las promociones o ascensos es una cuestión mediante la cual las empresas se consolidan en el área de la construcción. El desarrollo del equipo al interior facilita las relaciones interpersonales al comprender el impacto de las acciones personales sobre el éxito de las acciones de los demás miembros del grupo, al crear adhesión, fidelidad y compromiso de trabajo. Cuando se intento determinar la utilización de la competencia desarrollo del equipo, casi $82 \%$ de las constructoras, tienen una respuesta razonable sobre el particular. Número por demás elevado, que permiten inferir que existe preocupación por elevar la calidad del personal de 
las compañías constructoras. Al desagregarlas se detectó 39,68\% siempre apoyan al equipo y $41.27 \%$ frecuentemente.

\section{Competencia trabajo en equipo}

La capacidad de responder cuando se solicita a los responsables de obras o de gabinete de las constructoras a colaborar en equipo, dejando de lado las diferencias de orden personal para alcanzar un objetivo determinado o cumplir en tiempo y forma con una obra civil, un proyecto o un diseño, es básico para quien es el responsable de ejecutarlo. La cooperación y colaboración genuinas, en donde se antepone sobre cualquier cosa el logro de los objetivos y metas compartidos. El obtener resultados se vuelve en la piedra angular de la integración del equipo. No importan los sacrificios que deban hacerse en aras de cumplir con el cometido previsto. Los resultados reflejan que $70 \%$ contestó de forma conveniente. Al identificar el porcentaje de cuantas empresas se manifestaron por trabajar siempre en equipo fueron $38.10 \%$ y frecuentemente 31.75 por ciento.

\section{Competencias de logro y acción}

La promoción de actitudes y comportamientos orientados a conseguir objetivos de largo alcance dentro de la organización, al trabajar duro en ambientes cambiantes, en lapsos cortos y jornadas prolongadas, con miras a tener mayor productividad. Interactúan estrechamente para cumplir la misión y visón de cada empresa. Aquí, hay una razón de ser del empresario: ser emprendedor. El estudio demuestra que la mayor parte de los entrevistados tiene esta característica, pues casi $80 \%$ es emprendedor nato. Siempre tener esta característica lo constituye $55.56 \%$. Revelador, sin duda, puesto que uno de cada dos personas trabadoras de la industria utiliza siempre su habilidad negociadora para cumplir con los compromisos industriales adquiridos. Para reforzar lo anterior, $30.16 \%$ asume que frecuentemente utiliza esta postura.

\section{Competencia dinamismo-energía}

El empeño puesto en cada acción ejecutada, las ganas impuestas para conseguir los propósitos de la organización conlleva a la participación efectiva de sus integrantes, al trabajar duro en ambientes cambiantes o alternativos, con interlocutores diversos, que cambian en lapsos cortos, en jornadas prolongadas, sin afectar su productividad para cumplir con las cargas de trabajo previstas. De ello depende la eficacia de las tareas llevadas a cuestas. Esta competencia es la más desarrollada en sector de la 
construcción la conforma más de $82 \%$. $58.73 \%$ señaló siempre usar la competencia dinamismo-energía y $22.22 \%$ sostuvo usarla frecuentemente.

\section{Competencia iniciativa}

La decisión de incursionar en un mercado competido, es básica para un empresario. Estar dispuesto a actuar proactivamente para acordar qué hacer en el futuro inmediato. Establecer el rumbo con acciones concretas o cumplir aquellas propuestas en el pasado, e incluso la búsqueda de soluciones posibles en la empresa, es el signo que define a la competencia. Por ejemplo, determinar qué hacer para mejorar la operación o identificar a cuál ramo industrial dirigir los esfuerzos personales para conformar una industria es la característica propia del emprendedor. La iniciativa detectada ascendió a casi $67 \%$ de los casos estudiados en la muestra, desagregada siempre contestaron $28.57 \%$ y frecuentemente 38.10 por ciento.

Es importante resaltar que $58.73 \%$ declaró usar siempre la competencia dinamismoenergía en sus empresas. En contrapartida sólo $28.57 \%$ indicó usar siempre la iniciativa para efectuar sus labores de dirección en sus centros de trabajo. Para los industriales de la construcción tiene mayor relevancia aplicar el dinamismo-energía que iniciativa en los proyectos planteados.

\section{Competencia orientación de resultados}

La razón de existir de una organización es conseguir resultados óptimos en su operación. El hecho de contribuir a satisfacer alguna demanda social hace posible buscar los mejores métodos al alcance para lograrla. La rapidez aplicada y sentido de urgencia en la toma de decisiones encaminada a superar los objetivos y metas previstos, la capacidad de atender al cliente o mejorar la administración, al saber aplicar los procesos de producción sin que interfieran con los resultados, permite obtener frutos con un máximo de beneficio. De este modo, casi $86 \%$ de las empresas afirmó estar consciente de buscar orientación a resultados. Disociado se tiene que 55,56\% siempre está en la búsqueda de alcances para las compañías y $30.16 \%$ frecuentemente anda por ese mismo sendero.

\section{Competencias cognitivas}

Comprender la tendencia del entorno para tener una adecuada toma de decisiones, es una tarea que requiere tener al máximo las capacidades de reconocimiento para desarrollar estrategias de trabajo encaminadas a mejorar el rendimiento del recurso 
humano de las constructoras. De ahí el valor intrínseco de ejecutar acciones adecuadas, una vez analizado y entendido el medio donde se desarrollaran. Ello permite acertar en la orientación y curso de la empresa. La disyuntiva de poner en perspectiva el accionar tiene como consecuencia consolidar a la empresa en el mercado.

\section{Competencia pensamiento estratégico}

El análisis dentro y fuera de la organización constituye una pieza clave para el diseño de las políticas empresariales. La comprensión clara del movimiento del mercado es la base para encaminar los esfuerzos de la organización. La fortaleza, oportunidades, debilidades y amenazas (FODA), deben ser identificadas con claridad para rediseñar la estrategia empresarial. Implica conocer el entorno en el cual se desenvuelve la organización. Así, el estudio demostró que más de $83 \%$ aplica la competencia de pensamiento estratégico de comporta de una manera aceptable. De manera particular $49,21 \%$ la aplica siempre y $33.33 \%$ frecuentemente

\section{Competencias de ayuda y servicio}

Canalizar las necesidades y peticiones de los clientes para tenerlos satisfechos es el propósito de toda compañía. Al satisfacerlos seguirán adquiriendo los productos y servicios que proporcionan las empresas constructoras. El sentido de atención como queda establecido redundara en beneficio para las constructoras, al garantizar el trabajo y productos que ofrece el sector. De esta manera en la encuesta se observó que de forma general más de $76 \%$ utiliza las competencias de ayuda y servicio, al desagregarlas se tiene que $5 \mathrm{I} .59 \%$ siempre las utiliza y $24.60 \%$ frecuentemente las usa.

\section{Competencia orientación al cliente}

El servicio y atención al cliente constituye un engranaje que debe considerarse en el conjunto de las competencias. Se cifra en el hecho de servir y ayudar a los clientes tanto internos como externos, en conocer y resolver sus problemas relacionados con la adquisición de bienes o servicio producidos por la empresa. Es un componente que debe tenerse en cuenta si se pretende llegar a niveles superiores de atención en donde el cliente es el beneficiario final del esfuerzo organizacional. Principio y fin en la actuación del conjunto de actividades de los integrantes de la empresa para conseguir transacciones exitosas. Política que aplica la industria en alrededor de $75 \%$, que segregado lo utiliza siempre 47,62 y $26.98 \%$ lo usan con frecuencia. 


\section{Modalidad de contacto personal}

"En la forma de pedir, está la forma de dar" reza por hay un viejo proverbio mexicano. La manera establecer un ambiente de trabajo adecuado mediante la forma de interactuar con subalternos y personal de nivel jerárquico superior redunda habitualmente en éxito industrial. Contagiar de ánimo y mantener relaciones cordiales es una actitud que algunos líderes de organizaciones se empeñan en llevar a la práctica.También, es la forma de alentar a otros a compartir información y valorar la contribución de los demás. Sin embargo, es una competencia imprescindible dentro del conjunto de actividades desplegadas por las empresas. El contacto personal en las empresas constructoras de forma general es cercano a $78 \%$, nivel hasta cierto punto elevado, en virtud de que 8 de cada 10 dirigentes tienen un manejo adecuado con los distintos niveles jerárquicos. Al separarlos en el instrumento de medición siempre contestaron $55.56 \%$ de los encuestados y frecuentemente 22.22 por ciento.

\section{Competencias de eficacia personal}

Cambiar o mantenerse inmóvil frente a las variaciones generadas por el entorno es el dilema común que deben afrontar las empresas en un mercado competido. Las compañías constructoras no son ajenas a esta circunstancia. Para subsistir o más aun para acrecentar su participación en el sector, deben realizar todas aquellas acciones que conduzcan a mantenerse activas y con cierto margen de maniobra que les permita movilizar a la organización hacia nichos inexplorados que proporcionen estabilidad y garanticen permanencia en la industria. Inhibir la dinámica necesariamente conduce a la parálisis e inmovilismo, en consecuencia, provocan disminución de actividades y, en última instancia la quiebra y cierre de empresas. La constitución de un negocio tiene esa orientación, se asume a condición de ser perenne y guía fundamental de su conformación. El competir por un pedazo de mercado marca la carrera para avizorar y comprender el comportamiento de su evolución en aras de anticiparse y proponer los cambios requeridos para estar vigentes.

\section{Competencia habilidad para el cambio}

Considerarse apto y con la sapiencia suficiente para encontrar el camino por donde dirigir los esfuerzos empresariales, es un hecho que permite vaticinar el rumbo que debe tener la compañía frente a sus competidores. Es la aptitud para comunicar la visión de la estrategia a los órganos decisorios de la empresa, en donde se sustenta y transmite la misión y visión empresariales; en donde se vislumbra no tan solo como 
posible sino asequible, con la capacidad para crear compromisos genuinos tendientes a transformar la organización, con la intención de responder a las exigencias del entorno. Esa habilidad es una parte sustancial para garantizar el cambio que debe hacerse, con la asunción de los riesgos implícitos en las decisiones adoptadas. Así, el uso de la competencia habilidad para el cambio puede considerarse aceptable en su conjunto y ascendió a más de $65 \%$. En particular se utiliza siempre en $26.98 \%$ y frecuentemente 38.10 por ciento.

\section{Competencias de influencia}

Incidir en la toma de decisiones derivado de cuestiones subyacentes en los individuos de orden moral, reviste especial interés en la evaluación del estudio, debido a la escasa o nula importancia otorgada al comportamiento de los empleados en el ejercicio de sus funciones. Conceder poco valor a los actos derivados de la corrupción, propicia un entorno que intoxica el desarrollo de las actividades en el seno de la organización. Permea prácticamente todos sus niveles, desde las categorías superiores (directivos) hasta los de menor rango. Ello incide, sin duda en la calidad de las obras de construcción porque tienen un impacto directo en el tipo de material utilizado en la edificación, por ejemplo. Lo mismo sucede si hay cambio en las especificaciones técnicas que deben emplearse en el armado del acero de refuerzo para soportar construcciones pesadas o, si la mezcla de materiales para obtener concreto es diferente a las especificaciones técnicas aprobadas en el proyecto, entre otros. Los actos de corrupción, además de inmorales son perniciosos para la calidad de las obras, en detrimento de las construcciones y del prestigio empresarial de las constructoras. La dadiva entregada alguien debe pagarla y ese pago lo realiza el consumidor final de los inmuebles construidos con deficiencias.

\section{Competencia integridad}

El ser una persona cabal tiene un impacto positivo en las empresas. Aumenta la credibilidad en las acciones efectuadas horizontal y verticalmente, de arriba a abajo. Dota a la unidad empresarial de una cualidad que al paso del tiempo se hace inquebrantable. Es la capacidad de actuar en consonancia con lo que se dice y se hace, incluye la transmisión directa y franca de ideas, sentimientos e intenciones, incluso en situaciones difíciles con personas externas. Así, más de $68 \%$ de las empresas declararon utilizar la competencia referida a la integridad, considerada más o menos de uso periódico. De manera específica, el sector la utiliza siempre en 31.75\% y frecuentemente las usan $36.5 \mathrm{I}$ por ciento. 
De manera conclusiva puede esgrimirse que las empresas constructoras, en su conjunto, tienen un óptimo de operación -si se puede considerar así- que apenas ronda $70 \%$ de su capacidad productiva. Significa tener subutilizado $30 \%$ de toda la magnitud empresarial instalada, de forma permanente en cada obra o proyecto ejecutado. Situación de suyo insostenible que no debe seguir igual, si efectivamente quieren lograr mejores resultados. Deben hacerse esfuerzos por revertir esa situación, básicamente a través de un adecuado control de toda la gestión administrativa que desempeñan, independientemente de la perspectiva que muestran los resultados del estudio. A todas luces los constructores deben estar alertas para gestar los cambios organizacionales requeridos en aras de obtener el nivel óptimo de operación. La gestión de recursos humanos por competencias laborales ofrece una alternativa viable para disminuir la brecha negativa de productividad reflejada a lo largo de toda la investigación. Cierto es que implica el compromiso de comprender $y$, más aun capacitarse en el manejo del sistema, para ponerlo en marcha a la brevedad posible. Cada compañía puede determinar las competencias que debe aprehender en un sentido holístico, en donde el compromiso por el cambio que aportan sea entendido a cabalidad; la comprensión sobre el impacto positivo en su accionar redunde en beneficios inmediatos para las compañías. Y lo más importante, sean considerados genuinamente por los dueños y directivos, en quienes finalmente recaen las decisiones, políticas y estrategias fundamentales de la organización. No debe ser avizorada como un requisito burocrático más que los administradores han inventado para darle mayor complejidad, a las de por si complicadas actividades involucradas en el ámbito de la construcción. Ello conlleva a que los responsables y dueños de las empresas del sector comprendan por qué empresas internacionales se llevan las principales obras que además de realizarse en el Distrito Federal, también, se ejecutan en el interior de la Republica. La apuesta debe ser seria y consistente. Debe evitar quedarse cruzados de brazos y ver como constructoras internacionales se adueñan de negocios nacionalidades.

La repercusión en términos monetarios consiste en que solo obtienen 70 pesos de cada 100 posibles. A todas luces resulta técnica y financieramente insostenible, porque al hacer una proyección de la inversión utilizada en el ramo, se pierden alrededor de varios millones de pesos que traducidos, al interior de cada organización hay una pérdida neta irreparable si se sitúa en una perspectiva de largo plazo.

\section{Conclusiones y perspectivas}

El hecho de hacer recomendaciones sobre la forma de controlar y, aun más determinar los elementos que constituyen el entorno administrativo, en el cual las empre- 
sas pertenecientes a la industria de la construcción, debieran dirigir sus esfuerzos, resulta hasta cierto punto difícil porque no existe una receta única que pudiera llevarse a cabo para ser exitosos. Cada empresa tiene su manera de administrar que, en la mayoría de los casos puede considerarse fructífera. No obstante, es menester profundizar en el manejo de personal que ejecuta labores de campo o gabinete, en aras de obtener mayores niveles de productividad $y$, por ende, de ingresos que finalmente es el objetivo de toda organización. Así, al ceñirnos a los resultados del estudio, quizás las siguientes sugerencias pudieran ser de utilidad.

La gestión por competencias laborales a nivel directivo en la industria, en términos generales se utiliza alrededor de $73 \%$, quiere decir que 7 de cada 10 empresas las emplea al ejecutar sus labores. No obstante, en promedio (30.79\%), la mayoría de las compañías usan a veces las competencias. Es de notar que las empresas constructoras en el Distrito Federal, no están acostumbradas a aplicar la gestión por competencias laborales. Deben poner énfasis en orientar sus capacidades para implantar a la brevedad un sistema cuando menos con las competencias indicadas en el estudio, que les permita tener la posibilidad de obtener mayores ingresos $y$ consolidarse en el mercado.

Cierto es que existe una crisis financiera de orden mundial que afecta a la economía en su conjunto y en especial al sector de la construcción, que en apariencia no da visos de tener salida pronta de recuperación duradera. El hecho tiene relevancia para los constructores por la circunstancia de que deben afrontar problemas derivados de un contexto exógeno, que escapa a las soluciones inmediatas e internas a la empresa; pero deben escoger alternativas imaginativas, asumiendo disyuntivas que permitan aminorar los efectos de la crisis. El implantar un esquema de gestión por competencias laborales en las empresas se orienta en ese sentido. Redefinir su misión, visión y objetivos, así como alinearlos a éste sistema tendrá como consecuencia determinar las funciones y tareas asignadas de una forma más clara y transparente que les permite identificar con claridad qué esperan de la compañía y cómo pueden contribuir para mejorar su presencia y consolidación.

La propuesta se basa en el hecho de que son las competencias que están por encima de $80 \%$. Las más utilizadas por la industria. La competencia integridad está por debajo de $80 \%$, pero se propone debido a que debe estar presente en las compañías constructoras porque tiene efecto inmediato, al reducir la corrupción en la empresa y disminuir conductas contrarias a la Misión y Visión institucional.

Es de vital importancia que las empresas se dediquen a especializarse en alguna de las actividades de la construcción. Estar demasiado diversificadas a convierte en "aprendices de todo y maestros de nada". El potencial que representa la especialización actualmente constituye la pieza angular de los negocios a nivel mundial, sin 
que signifique dejar la posibilidad de complementarse con otros productos; es decir, deben asegurar tener una actividad principal o "estrella" y, al mismo tiempo, dos o tres complementarias a la función fundamental. Conviene suponer que si se dedican a la construcción de carreteras, como función principal, entonces como complementaria podría ser el mantenimiento de las mismas o la construcción de puentes.

Del estudio también, se desprende que el personal de dirección tiene escasa comprensión y sensibilidad respecto a la rentabilidad que tendría la industria al manejarse en alguna área específica de la construcción e ignora la importancia que, implica la decisión de posesionarse de un determinado nicho mercado a través de un cierto producto o servicio específico, como los indicados con anterioridad. Al dejarlo de hacer o postergarlo para tiempos mejores, inhiben su capacidad emprendedora, con el consecuente retraso en la mejora de resultados. Tiene que revertirse esa tendencia y dirigir sus esfuerzos a romper inercias y buscar la eficacia empresarial, porque a final de cuentas el propósito de iniciar una firma, es mantenerse en un entorno por difícil y azaroso que sea y, más aun perpetuarse indefinidamente. El riesgo de apostar capital en empresas endebles o poco efectivas no es el fin de los inversionistas por excéntricos que sean. No. Su finalidad es muy clara: obtener el máximo de dividendos por la suma de dinero colocada en cada proyecto, independientemente del desembolso que implique. Por tanto debe asumirse la posición de explotar una función principal al máximo y algunas otras de forma complementaria, lo cual redundará en el mediano y largo plazo en una mayor penetración y consolidación en el mercado.

Evidentemente es preponderante el número de micro y pequeñas empresas que, en su mayoría se dedican a diversas actividades de la construcción, en vez de prepararse para una actividad en específico, quizá el llamado de atención sería para ellas, deberían pensar en la manera de especializarse a fin de ser más rentables y productivas. Con ello, tendrían la posibilidad de tener mayor presencia en el sector de la construcción.

De lo anterior puede decirse que las empresas deben otorgar mayor importancia a las competencias laborales cognoscitivas como es el caso del pensamiento estratégico que traducido a la empresa constituye la Visión del negocio, en donde su orientación no es precisamente un conjunto de teorías sobre el manejo empresarial, sino el punto de vista del estratega de los negocios que ve el mundo de cierta manera, debido a que observa las actividades de los negocios tal como se desarrollan en el mercado competitivo. La constante del pensamiento estratégico es la posibilidad de cambiar de idea para adaptarse a requerimientos del medio donde se desarrolla el negocio, por ende, expresa un conjunto de "intenciones" en forma de un plan estratégico. El cual frecuentemente, se desarrolla como un enunciado de la visión 
que la empresa tiene acerca del alcance de sus operaciones, de sus metas, objetivos y programas para lograr el éxito dentro del contexto del ambiente competitivo previsto.

El estudio cumple con el cometido de trabajo al determinar que en promedio se entrevistó a empresas que tuvieron más de 30 empleados, correspondientes a pequeñas empresas constructoras. Prácticamente, se cubrió $94.7 \%$ de las industrias registradas en el SIEM, si se considera a las micro industrias.

Es sabido que en estudios estadísticos existen diferentes formas de calcular las medidas de tendencia central de una muestra, pueden ser: Media Aritmética, Mediana, Media Geométrica, Media Armónica o Moda estadística. En el análisis de la información, se observó que las variables esencialmente son de orden cualitativo; por tanto, se opto por el uso de la moda estadística. La moda se define como aquel valor de la variable al que corresponde máxima frecuencia, en este caso, es relativa para una serie de datos agrupados. Se determinó que la mayor frecuencia, era el análisis pertinente y representativo para los fines de la investigación, es decir, el valor mayor relativo, el cual está en el pico más alto de la distribución. Las competencias observadas y algunas otras actividades administrativas estuvieron calculadas mediante esta medida de tendencia central. En menor medida se utilizó la Media Aritmética, en particular para determinar las personas ocupadas en el sector. De modo que las inferencias realizadas obedecen esencialmente a estas dos medidas de tendencia central. 